\title{
ACCESSIBLE SPECTRUM ANALYSER
}

\author{
Fiore Martin, Oussama Metatla, Nick Bryan-Kinns and Tony Stockman
}

\author{
Centre For Digital Music \\ School of Electronic Engineering and Computer Science \\ Queen Mary University of London \\ London, E1 4NS \\ UK \\ t.stockman@qmul.ac.uk
}

\begin{abstract}
This paper presents the Accessible Spectrum Analyser (ASA) developed as part of the DePic project (Design Patterns for Inclusive collaboration) at Queen Mary University of London. The ASA uses sonification to provide an accessible representation of frequency spectra to visually impaired audio engineers. The software is free and open source and is distributed as a VST plug-in under OSX and Windows. The aim of reporting this work at the ICAD 2016 conference is to solicit feedback about the design of the present tool and its more generalized counterpart, as well as to invite ideas for other possible applications where it is thought that auditory spectral analysis may be useful, for example in situations where line of sight is not always possible.
\end{abstract}

\section{INTRODUCTION}

The Design Patterns for Inclusive Collaboration (DePIC) project aimed to develop new ways for people to interact with each other using different senses, so reducing barriers caused by visual and other sensory impairments (depic.eecs.qmul.ac.uk).

The development of the Accessible Spectrum Analyser, ASA, came out of our collaboration with visually impaired (VI) audio engineers and musicians, some of the results of which, including the development and evaluation of an Accessible Peak-level Meter, APM, were reported at the ICAD 2015 conference [1]. The requirement for the ASA came as a result of VI audio engineers explaining the need for an accessible means of examining the power in specific frequency bands of the audio signal being edited. However, this is only one possible application area for an ASA. Visually impaired school students studying GCSE and higher level Physics, electronics and engineering courses have the need to be able to view spectra of various kinds. Clearly spectral analysis, as a technique, is applied in numerous scientific and engineering applications. We are planning to develop a more generalized version of the tool for use as a sensory substitution device in science and engineering education and practice. Furthermore, there may be potential for applications of the tool by sighted users who need to monitor spectra without having line of sight to the visual display.

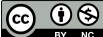

This work is licensed under Creative Commons Attribution Non Commercial 4.0 International License. The full terms of the License are available at http://creativecommons.org/licenses/by-nc/4.0
The ASA we have developed is a real-time spectrum analysis plug-in that allows visually impaired users to examine spectrograms using data sonification. The aim of reporting this work at the ICAD conference is to solicit feedback about the design of the present tool and its more generalized counterpart, as well as to invite ideas for other possible applications where it is thought that auditory spectral analysis may be useful.

\section{SONIFICATION DESIGN}

The sonic representation of spectrograms in this software is primarily designed to enable the user to monitor a specific frequency band to determine when, and to what extent power in that band exceeds a user-specified threshold.

The sonification uses a pitch mapping with an up-up polarity, that is, the higher the pitch the higher the peak level of power within the chosen frequency band. The user specifies the frequency range to be monitored, and sets the threshold level in $\mathrm{dB}$, and, as soon as the energy of any frequency in the selected frequency band goes past the threshold, the ASA sounds a short beep. The beep starts at $440 \mathrm{~Hz}$ and it is raised one semitone for each $\mathrm{dB}$ of difference between the frequency magnitude and the user specified threshold. In case more than one frequency within the selection is higher than the threshold, the highest one is represented in the sonification.

If the ASA is used to monitor a stereo signal, the two channels are mixed together before being analysed. In the ASA, the sonification is panned from left to right, where the pan position represents the location in the spectrum of the peak frequency with respect to the whole spectrum, ranging from $20 \mathrm{~Hz}$ on the very left, to $20050 \mathrm{~Hz}$ on the very right. For example, if the selected spectrum peaks at $50 \mathrm{~Hz}$, then the beep is presented towards the left, whereas if the peak is at $20 \mathrm{kHz}$, the beep will occur towards the right.

It has been helpfully pointed out by a reviewer of this submission that parameter mappings which make use of panning must be used with care. It restricts the end use cases to where a listener is positioned within the sweet spot, or tethered to a DAW via headphones. This will need to be borne in mind if we wish to optimize the mapping for situations where 'line-of-sight' is compromised, because in that case panning may fail to produce a meaningful display for the listener, if they are not facing the stereo speaker array or are too far away for headphones. One solution may be to encode the data redundantly with another parameter. For example the rate 
of beeping could increase as frequency peaks approach the upper end of the spectrum.

\section{PARAMETERS}

The plug-in comes with five tweakable parameters:

1. Threshold: sets the threshold in $\mathrm{dB}$ for the spectral sonification. If any frequency within the selection is higher than the threshold, then the plug-in will emit a beep;

2. Selection Start: sets the starting point, in Hertz, of the selection. Frequencies within the selection will be monitored for peaks;

3. Selection Size: sets the size of the selection, from the starting point. For example, if the selection starts at $1000 \mathrm{~Hz}$ and the selection size is $500 \mathrm{hz}$, then all the frequencies between 1000 and $1500 \mathrm{~Hz}$ will be monitored for peaks;

4. Dry: controls the level of the input audio, namely the audio content to be analyzed;

5. Wet: controls the level of the sonification.

The Accessible Spectrum Analyser provides access to the parameters by exposing them to inspectors - such as the ReaAccess plug-in (used with the Reaper DAW) or the Cakewalk Sonar inspector - in a clear and well formatted way.

\section{EVALUATION}

Because the development of the ASA came at the very end of the DEPIC project that funded it, a full evaluation of the tool has not so far been possible. However, information about the plug-in has been widely distributed on email lists for visually impaired musicians and audio engineers. The take up of the tool has been fairly limited, but those musicians and audio engineers who have adopted it have been very enthusiastic about its functionality, citing the fact there are currently no similar tools that provide direct access to signal spectra through audio currently. The few criticisms that have been received have reflected the reviewer feedback described above, that the panning functionality is not welcomed by all users.

The accessible spectrum analyser was downloaded a total of 109 times from outside the Queen Mary campus in February and March. The bulk of these downloads occurred in March, after the announcement of the plug-ins release on relevant forums and mailing lists at the end of February. This provides unspectacular but solid evidence of the demand for this early-stage version, which has so far only been advertised to one subset of the possible target population.

\section{FUTURE WORK}

As mentioned above, we hope to develop a more functional and flexible version of the ASA, capable of providing auditory access to other commonly used signal measures, such as auto and cross correlation, coherence, cepstrum etcetera. To this end, we will investigate currently available open source, freely available packages for signal analysis, such as Octave or those available from Physionet, to provide a signal analysis engine on which to base a more general accessible signal analysis front-end.

\section{OBTAINING THE SOFTWARE}

The binaries for the ASA software, along with other software releases from the DEPIC project, can be downloaded from [2]. This work is licensed under the Creative Commons Attribution Non Commercial 4.0 International License. The full terms of the License are available at [3]. The source code of the Accessible Spectrum Analyser is available in the SoundSoftware repository, located at [4]. It is released under the Cokos WDL license, which in short means you can alter it and redistribute it freely, even without providing the source code of your derivative work.

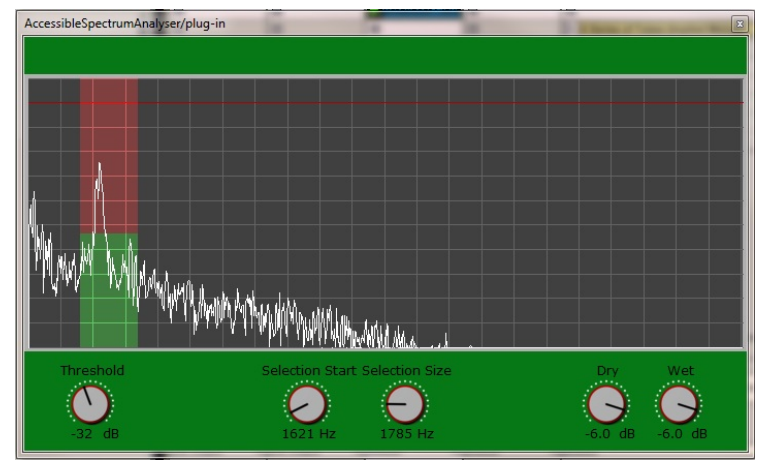

(a) Spectrum Analyser

\section{REFERENCES}

[1] Metatla, O. Bryan-Kinns, N. Stockman, T and Martin, F. Sonifications for Digital Audio Workstations: Reflections on a Participatory Design Approach, Proceedings of ICAD 2015.

[2] http://depic.eecs.qmul.ac.uk/?q=software

[3] http://creativecommons.org/licenses/by-nc/4.0

[4] https://code.soundsoftware.ac.uk/projects/asa 